\title{
HIROTA DERIVATIVES AND REPRESENTATION THEORY
}

\author{
CHRIS ATHORNE \\ Department of Mathematics, University of Glasgow, Glasgow G12 8QW, UK \\ e-mail: ca@maths.gla.ac.uk
}

(Received 14 March, 2000)

\begin{abstract}
It is shown that the Hirota derivative can be used to construct the plethysm for tensor products of representations of $\mathfrak{S l}_{2}(k)$.
\end{abstract}

1991 Mathematics Subject Classification. 35Q51, 17B80.

1. Introduction. The Hirota 'derivative' [5] is a process of considerable power in the theory of solitons and integrable systems generally. Its use allows the representation of many integrable equations in bilinear form and thereby simplifies the analytic process of the inverse scattering transform [6] to an algebraic procedure for a large class of solutions. It retains nevertheless a certain mystique. The aim of this paper is to unveil the Hirota derivative, just a fraction, by exploring its connection with representation theory $[\mathbf{1 , 9}]$.

We start by formulating the Hirota derivative as a map of tensor products of $\mathfrak{S I}_{2}(k)$-modules, where $k$ is any field of zero characteristic, and showing that it acts as a partial intertwining operator for the action of this algebra. The main thrust of the paper is to show that the entire decomposition of the tensor product into irreducible representations can be accomplished by applying the Hirota map to a specific lowest weight vector. This is achieved by creating a new $\mathfrak{S} \mathfrak{l}_{2}(k)$-module, a homogeneous, graded algebra, to which standard results can be applied. On the way certain interesting Hilbert-Poincaré series are presented.

2. The Hirota derivative. Let $k$ be a field of characteristic 0 and denote by $e, f$ and $h$ the $k$-basis of the algebra $\mathfrak{S l}_{2}(k)$ satisfying the Lie product identities:

$$
[e, f]=h, \quad[h, e]=2 e, \quad[h, f]=-2 f .
$$

By the $\mathfrak{S l}_{2}(k)$-module $\mathcal{A}_{n}$ we mean the standard $n+1$ dimensional representation of $\mathfrak{S I}_{2}(k)$ with basis $\left\{a_{0}, a_{1}, \ldots, a_{n}\right\}$ with respect to which $e, f$ and $h$ may be taken to be differential operators:

$$
\begin{gathered}
e=n a_{1} \frac{\partial}{\partial a_{0}}+(n-1) a_{2} \frac{\partial}{\partial a_{1}}+\ldots+a_{n} \frac{\partial}{\partial a_{n-1}}, \\
f=a_{0} \frac{\partial}{\partial a_{1}}+2 a_{1} \frac{\partial}{\partial a_{2}}+\ldots+n a_{n-1} \frac{\partial}{\partial a_{n}}, \\
h=n a_{n} \frac{\partial}{\partial a_{n}}+(n-2) a_{n-1} \frac{\partial}{\partial a_{n-1}}+\ldots-n a_{0} \frac{\partial}{\partial a_{0}} .
\end{gathered}
$$


Let $\mathcal{A}_{n}^{g}$ be the $g$-fold $k$-tensor product of $\mathcal{A}_{n}$. We shall write elements of this tensor product as $k$-linear combinations of lists of $g$ integers (between 0 and $n$ ) under the correspondence:

$$
a_{k_{1}} \otimes a_{k_{2}} \otimes \ldots \otimes a_{k_{g}} \leftrightarrow\left(k_{1}, k_{2}, \ldots, k_{g}\right)
$$

The operators $e, f$ and $h$ are taken to extend to $\mathcal{A}_{n}^{g}$ in the usual fashion (Leibnitz rule). $\mathcal{A}_{n}^{g}$ decomposes as a direct sum of eigenspaces for $h$. The associated eigenvalues are the weights.

Definition 1. The isobaric weight of $\left(k_{1}, \ldots, k_{g}\right) \in \mathcal{A}_{n}^{g}$ is $\left.p=k_{1}+k_{2}+\ldots+k_{g}\right)$. The weight of this element is $2 p-n g$.

The effect of $e(f)$ is to increase (decrease) the isobaric weight of an isobaric element by one and hence the weight by two.

We now introduce the key player.

Definition 2. The Hirota map $D_{i j}: \mathcal{A}_{n}^{g} \rightarrow \mathcal{A}_{n+1}^{g}$ is defined by $k$-linear extension of its action on a basis, an action given by:

$$
D_{i j}\left(\ldots, k_{i}, \ldots, k_{j} \ldots\right)=\left(\ldots, k_{i}+1, \ldots, k_{j} \ldots\right)-\left(\ldots, k_{i}, \ldots, k_{j}+1 \ldots\right)
$$

Note that there are only $g-1$ linearly independent $D_{i j}$ and that the map is antisymmetric in its two indices. $D_{i j}$ increases the isobaric weight of an isobaric element by one but the weight by $2-g$.

The original form of the Hirota map ('derivative') [5] appears in the theory of soliton equations where its remarkable properties make it of prime importance to the explicit description of soliton solutions [8]. The definition given above is actually associated with a generalized Hirota derivative [3] though the current form of its presentation is due to [1]. The link to algebraic invariants, and hence representation theory, is amplified in [1] and remarked upon in [9].

From our point of view the most important property of the Hirota map is that it is a partial intertwining operator for the $\mathfrak{S l}_{2}(k)$-modules $\mathcal{A}_{n}^{g}$ and $\mathcal{A}_{n+1}^{g}$. This may be expressed precisely as follows.

Lemma 3. The maps $D_{i j}: \mathcal{A}_{n}^{g} \rightarrow \mathcal{A}_{n+1}^{g}$ satisfy the following commutation rules with $f$ and $h$ :

$$
\begin{aligned}
& {\left[D_{i j}, f\right]=0,} \\
& {\left[D_{i j}, h\right]=(g-2) D_{i j} .}
\end{aligned}
$$

Note that in the lemma $f$ and $h$ act on both $\mathcal{A}_{n}^{g}$ and $\mathcal{A}_{n+1}^{g}$. There appears to be no simple expression for the commutator of $D_{i j}$ with $e$.

The chief consequence of the partial intertwining relations is that elements of $\mathcal{A}_{n}^{g}$ which are killed by $f$, that is lowest weight vectors, are mapped by the $D_{i j}$ into lowest weight vectors in $\mathcal{A}_{n+1}^{g}$. Since we know that $\mathcal{A}_{n}^{g}$ decomposes into irreducible representations of $\mathfrak{g} \mathfrak{l}_{2}(k)$ the Hirota map will generate associated lowest weight vectors from the most basic one $(0, \ldots, 0) \in \mathcal{A}_{n}^{g}$. The question which we want to address is 
whether it generates the lowest weight vectors of all the irreducibles in $\mathcal{A}_{n}^{g}$. We shall show that the answer is yes by a counting argument. Before we do so, we give a couple of simple examples.

\section{Examples of plethysm.}

$\mathcal{A}_{2}^{3}$

The basic element $(0,0,0)$ has weight -6 and is the lowest weight vector for a 7 dimensional irreducible representation. Now consider $(0,0,0) \in \mathcal{A}_{1}^{3}$. There are two linearly independent highest weight vectors in $\mathcal{A}_{2}^{3}, D_{12}(0,0,0)=(1,0,0)-(0,1,0)$ and $D_{13}(0,0,0)=(1,0,0)-(0,0,1)$ each of weight -4 and giving 5 dimensional irreducible representations. For $(0,0,0) \in \mathcal{A}_{0}^{3}$ each of $D_{12}^{2}(0,0,0)=(2,0,0)-$ $2(1,1,0)+(0,2,0), D_{13}^{2}(0,0,0)=(2,0,0)-2(1,0,1)+(0,0,2)$ and $D_{12} D_{13}(0,0,0)=$ $(2,0,0)-(1,1,0)-(1,0,1)+(0,1,1)$ generate the 3 dimensional representations and finally $D_{12} D_{23} D_{31}(0,0,0)=-(2,1,0)-(1,0,2)+(2,0,1)-(0,2,1)+(1,2,0)+$ $(0,1,2)$ belongs to $\mathcal{A}_{2}^{3}$, as a subspace of $\mathcal{A}_{3}^{3}$, has weight 0 and is a one dimensional representation (invariant). These are all the lowest weight vectors which can be generated using the Hirota maps and it is easy to see that they give the complete decomposition by summing over dimensions with multiplicities:

$$
\mathbf{3} \otimes \mathbf{3} \otimes \mathbf{3}=\mathbf{7} \oplus \mathbf{5}^{2} \oplus \mathbf{3}^{3} \oplus \mathbf{1}
$$

$\mathcal{A}_{2}^{4}$

Briefly, we have a 9 dimensional representation with lowest weight vector $(0,0,0,0) \in \mathcal{A}_{2}^{4}$; three 7 dimensional representations coming from $D_{12}, D_{13}$ and $D_{14}$ acting on $(0,0,0,0) \in \mathcal{A}_{1}^{4}$; six 5 dimensional representations from $D_{12}^{2}, D_{13}^{2}, D_{14}^{2}$, $D_{12} D_{13}, D_{12} D_{14}$ and $D_{14} D_{13}$; six 3 dimensional representations coming from $D_{12}^{2} D_{34}$, $D_{12} D_{34}^{2}, D_{13} D_{24}^{2}, D_{13}^{2} D_{24}, D_{14} D_{32}^{2}$ and $D_{14}^{2} D_{32}$; and three 1 dimensional coming from $D_{12}^{2} D_{34}^{2}, D_{13}^{2} D_{24}^{2}$ and $D_{14}^{2} D_{32}^{2}$. This is precisely the decomposition,

$$
\mathbf{3} \otimes \mathbf{3} \otimes \mathbf{3} \otimes \mathbf{3}=\mathbf{9} \oplus \mathbf{7}^{3} \oplus \mathbf{5}^{6} \oplus \mathbf{3}^{6} \oplus \mathbf{1}^{3} .
$$

One may do many such examples by hand, reinforcing the conjecture that the Hirota map and the action of $e$ construct all the irreducibles in the tensor product.

4. Counting lowest weight vectors. If our conjecture is correct the lowest weight vectors in $\mathcal{A}_{n}^{g}$ will come from polynomials in the $D_{i j}$ which, when applied to $(0,0, \ldots, 0)$, never yield an integer greater than $n$. This is the same as seeking polynomials over $k$ in the differences $x_{i}-x_{j}$ of $g$ variables $x_{1}, \ldots, x_{g}$ which involve no power of any $x_{i}$ greater than $n$.

Let $R$ be the polynomial ring $k\left[x_{1}, \ldots, x_{g}\right], I$ the ideal of $R$ generated by the set $\left\{x_{1}^{n+1}, \ldots, x_{g}^{n+1}\right\}$ and $J$ the ideal of $R$ generated by the element $x_{1}+x_{2}+\ldots+x_{g}$. We have the short exact sequence:

$$
I \hookrightarrow R \rightarrow R / I .
$$

Let $\phi$ be the map, 


$$
\phi: R \rightarrow R, \quad x_{i} \rightarrow x_{i}-\frac{1}{g}\left(x_{1}+x_{2}+\ldots+x_{g}\right)
$$

which has kernel $J$ and let $\pi$ be the projection

$$
\pi: R \rightarrow R / J
$$

This is precisely the polynomial ring in the differences of the $x_{i}$. Now apply $\pi$ to (4). It is easy to see that we obtain the short exact sequence,

$$
\frac{I}{I \cap J} \hookrightarrow \frac{R}{J} \rightarrow \frac{(R / J)}{I /(I \cap J)}
$$

and, by the isomorphism theorems,

$$
\frac{(R / J)}{I /(I \cap J)} \cong \frac{(R / J)}{(I+J) / J} \cong \frac{R}{I+J}
$$

which is the homogeneous (finite dimensional) $k$-algebra

$$
K_{g}^{n}=\frac{R}{\left(x_{1}^{n+1}, \ldots x_{g}^{n+1}, x_{1}+x_{2}+. .+x_{g}\right) R} .
$$

This homogeneous algebra is graded by isobaric weight

$$
K_{g}^{n}=\bigoplus_{p=0}^{n g} K_{g}^{n, p}
$$

and the $k$-dimension of each component is the number of lowest weight vectors constructed by use of Hirota maps. (Note that for $p$ larger than some value between 0 and $n g$ these dimensions are zero.) Each comes with an irreducible representation of dimension $n g-2 p+1$ and so we have to show that

$$
\sum_{p=0}^{n g}(n g-2 p+1) \operatorname{dim} K_{g}^{n, p}=(n+1)^{g}
$$

which is the dimension of $A_{n}^{g}$. This is the same as showing that

$$
(n g+1) k_{g}^{n}(1)-2 k_{g}^{\prime n}(1)=(n+1)^{g}
$$

where $k_{g}^{n}(s)$ is the Hilbert-Poincaré series of $K_{g}^{n}$,

$$
k_{g}^{n}(s)=\sum_{p=0}^{n g} s^{p} \operatorname{dim} K_{g}^{n, p}
$$

and prime denotes the derivative with respect to $s$.

The assertion is relatively easily verified in particular instances using algorithms based on Gröbner bases implemented in software packages like MAPLE [7]. However, the proof is not so easily accomplished for general $n$ and $g$. 
5. The Hilbert-Poincaré Series. This section constitutes the meat of the present paper and is devoted to obtaining a closed form expression for the Hilbert-Poincare series, $k_{g}^{n}(s)$, of $K_{g}^{n}$. The algebra is constructed as the kernel of a differential operator acting on a simpler graded algebra. Let $h_{g}^{n}(s)$ denote the Poincaré-Hilbert series of the homogeneous algebra $R / I$ and let $\Delta$ denote the differential operator $\partial_{x_{1}}+\partial_{x_{2}}+\ldots \partial_{x_{g}}$.

Lemma 4. $\Delta$ maps $R / I$ to itself with kernel $R /(I+J)$.

Proof. This is a straightforward consequence of the theory of partial differential equations with constant coefficients.

Lemma 5. The isobaric weight gives a grading of $R / I$

$$
R / I=\bigoplus_{p=0}^{n g} M_{g}^{n, p}
$$

(every component of which is nontrivial) and $\Delta$ maps $M_{g}^{n, p}$ to $M_{g}^{n, p-1}$.

Proof. The grading is obvious and since the element $a=\left(x_{1} \ldots x_{g}\right)^{n}$ provides a basis for $M_{g}^{n, n g}$ and $\Delta^{n g} a=(n g) ! \neq 0$ we see that all the components are non trivial.

Lemma 6. The Hilbert-Poincaré series of $R / I$ is

$$
h_{g}^{n}(s)=\left(1+s+s^{2}+\ldots+s^{n}\right)^{g}
$$

Proof. By induction on $g$ :

$$
M_{g}^{n, p}=M_{g-1}^{n, p} \oplus x_{g} M_{g-1}^{n, p-1} \oplus x_{g}^{2} M_{g-2}^{n, p-2} \oplus \ldots \oplus x_{g}^{n} M_{g-1}^{n, p-n}
$$

and hence

$$
h_{g}^{n}(s)=h_{g-1}^{n}(s)+s h_{g-1}^{n}+s^{2} h_{g-1}^{n}+\ldots+s^{n} h_{g-1}^{n}
$$

and it is straightforward that

$$
h_{2}^{n}=1+s+s^{2}+\ldots+s^{n} .
$$

The idea now is to show that $R / I$ actually carries a representation of $\mathfrak{s} \mathfrak{l}_{2}(k)$ in which $K_{g}^{n}$ appears as the span of lowest weight vectors. We achieve this by means of the following constructions very loosely analogous to the Hodge star and coboundary operations in the theory of de Rham complexes.

Definition 7. Let $a$ be a monomial element of $R / I$. We define $* a$ to be the monomial with the property $a * a=\left(x_{1} x_{2} \ldots x_{g}\right)^{n}$. Now extend $*$ by $k$-linearity to the general element of $R / I$. $*$ thus maps $M_{g}^{n, p}$ to $M_{g}^{n, n g-p}$. 
It is easily seen, by the symmetry of the Hilbert-Poincare series,

$$
s^{n g} h_{g}^{n}(1 / s)=h_{g}^{n}(s)
$$

that $*$ is an isomorphism of $k$-vector spaces.

Definition 8. With the map $\Delta: M_{g}^{n, p} \rightarrow M_{g}^{n, p-1}$ we associate a "dual" map $\delta: M_{g}^{n, p} \rightarrow M_{g}^{n, p+1}$ defined by

$$
\delta=* \Delta *
$$

Lemma 9. The operators $\delta, \Delta$ and $H=\delta \Delta-\Delta \delta$ make $R / I$ into an $\mathfrak{S l}_{2}(k)$-module.

Proof. By considering the action of the operators on a monomial element it can easily be seen that:

$$
\begin{gathered}
\delta=n\left(x_{1}+\ldots+x_{g}\right)-x_{1}^{2} \partial_{1}-\ldots-x_{g}^{2} \partial_{g} \\
H=n g-2\left(x_{1} \partial_{1}+\ldots+x_{g} \partial_{g}\right) \\
{[H, \Delta]=2 \Delta} \\
{[H, \delta]=-2 \delta}
\end{gathered}
$$

Theorem 10. $R / I$ decomposes into a direct sum of irreducible $\mathfrak{S l}_{2}(k)$-modules isomorphic to $\mathcal{A}_{d}$ of dimensions $d=n g+1, n g-1, n g-3, \ldots$ with multiplicities

$$
\mu_{d}=\operatorname{dim} M_{g}^{n,(d+n g-1) / 2}-\operatorname{dim} M_{g}^{n,(d+n g+1) / 2} .
$$

Proof. This follows from standard representation theory [2]. The $M_{g}^{n, p}$ are the eigenspaces of $H$ with weights $n g-2 p$. Each lowest weight vector, $v$ with $\delta(v)=0$, of weight $n g-2 p$ belongs to an irreducible representation isomorphic to $\mathcal{A}_{d}$ of dimension $d=2 p-n g+1$. The highest dimensional irreducible is associated with the lowest weight vector $\left(x_{1} \ldots x_{g}\right)^{n} \in M_{g}^{n, n g}$ and has dimension $n g+1$. Factoring out this irreducible we are left with $\operatorname{dim} M_{g}^{n, n g-1}-\operatorname{dim} M_{g}^{n, n g}$ lowest weight vectors in $M_{g}^{n, n g-1}$ each generating irreducibles of dimension $n g-1$. Factoring out these and continuing we arrive at the result.

A consequence of this is that, because of the symmetry between highest and lowest weight vectors, we can easily construct the kernel of $\Delta$ as the span of the highest weight vectors and this gives the result:

TheOrem 11. The Hilbert-Poincaré series of $R /(I+J)$ is

$$
k_{g}^{n}(s)=\left[(1-s) h_{n}^{g}(s)\right]_{+}
$$

where $[\cdot]_{+}$denotes the truncation to the terms with positive coefficients. 
Finally, we see that the formula (6) for the total dimensions of all the irreducible representations generated by the Hirota maps is just same as the formula for the total dimension of all the irreducibles in $R / I$, that is the dimension of $R / I$. Hence:

THEOREM 12.

$$
(n g+1) k_{g}^{n}(1)-2 k_{g}^{\prime n}(1)=h_{g}^{n}(1)=(n+1)^{g} .
$$

The approach above immediately generalizes to the g-fold tensor product of $\mathfrak{S l}_{2}(k)$-modules of arbitrary dimensions:

$$
\mathcal{A}_{n_{1}, \ldots, n_{g}}^{g}=A_{n_{1}} \otimes A_{n_{2}} \otimes \ldots \otimes A_{n_{g}} .
$$

We therefore consider the homogeneous algebra

$$
R /\left(x_{1}^{n_{1}+1}, \ldots, x_{g}^{n_{g}+1}\right) R
$$

whose Hilbert-Poincaré polynomial is

$$
h^{n_{1}, n_{2}, \ldots, n_{g}}(s)=\prod_{i=1}^{g}\left(1+s+\ldots+s^{n_{i}}\right)
$$

and which carries a $\mathfrak{S l}_{2}(k)$-representation given by the differential operators $\Delta=\partial_{1}+\ldots \partial_{g}$ and $\delta=* \Delta *$, where $*$ is defined by its $k$-linear extension from its action on monomials $a$, namely,

$$
a(* a)=x_{1}^{n_{1}} x_{2}^{n_{2}} \ldots x_{g}^{n_{g}} .
$$

Explicitly, $\delta$ is given by

$$
\delta=n_{1} x_{1}+\ldots+n_{g} x_{g}-x_{1}^{2} \partial_{1}-\ldots-x_{g}^{2} \partial_{g}
$$

and

$$
H=[\Delta, \delta]=n_{1}+\ldots+n_{g}-2\left(x_{1} \partial_{1} \ldots x_{g} \partial_{g}\right) .
$$

$\Delta, \delta$ and $H$ are easily seen to satisfy the commutation relations of $\mathfrak{s l}_{2}(k)$. Again the Hilbert-Poincaré polynomial is $\left[(1-s) \prod_{i=1}^{g}\left(1+s+\ldots+s^{n_{i}}\right)\right]_{+}$and the entire decomposition arises from Hirota maps, because the dimensions satisfy the formula

$$
\left(n_{1}+\ldots+n_{g}+1\right) k(1)-2 k^{\prime}(1)=\prod_{i=1}^{g}\left(n_{i}+1\right) .
$$

6. Conclusion. We have presented a derivation of Hilbert-Poincaré series for certain homogeneous algebras generated by the generalized Hirota map and shown thereby that such maps generate the entire decomposition of tensor products of standard representations into irreducibles. This can be regarded as an extension of the constructive methods of classical invariant theory [9]. 
There are two obvious directions for further work.

Firstly, given the importance of the Hirota derivative in integrable systems theory, one may ask whether the above results indicate an 'explanation' for this enigmatic phenomenon. There are, for instance, connections with the Moyal bracket of quantum mechanics [10].

Secondly, to what extent can this simple and attractive algebraic structure be generalised to other Lie algebras or to differential operators other than $\Delta$; for example, operators of second order?

Finally, I should like to thank Ken Brown for one or two critical ears.

\section{REFERENCES}

1. C. Athorne, Algebraic invariants and generalized Hirota derivatives, Phys. Lett. A 256 (1999), 20-24.

2. W. Fulton and J. Harris, Representation theory (Springer-Verlag, 1991).

3. B. Grammaticos, A. Ramani and J. Hietarinta, Multilinear operators: the natural extension of Hirota's bilinear formalism, Phys. Lett. A 190 (1994), 65-70.

4. D. Hilbert, Lectures on algebraic invariants (Cambridge University Press, 1993). $323-331$

5. Ryogo Hirota, Bilinearization of soliton equations, J. Phys. Soc. Japan 51 (1982),

6. S. V. Manakov, S. Novikov, L. P. Pitaevskii and V. E. Zakharov, Theory of solitons (Plenum, 1984).

7. Maple V Release 5.00 (Waterloo Maple Inc., 1997).

8. J. J. C. Nimmo, Hirota's method, in Soliton theory: a survey of results, Nonlinear Science: Theory and Applications (Manchester University Press, Manchester, 1990), 75-96.

9. P. J. Olver, Classical invariant theory (Cambridge University Press, 1999).

10. I. A. B. Strachan, A geometry for multidimensional integrable systems, J. Geom. Phys. 21 (1997), 255-278. 